\title{
LA NARRATIVA ITALIANA Y LA PRIMERA GUERRA MUNDIAL: LA VOZ DE LAS ESCRITORAS
}

\author{
ITALIAN NARRATIVES AND THE FIRST WORLD WAR: THE WRITERS' VOICE
}

Laura Rosúa Aguilera

Universidad de Granada

\section{Resumen:}

A pesar de que el papel desempeñado por las mujeres en la sociedad del período de la Gran Guerra ha sido reconocido como fundamental por la historiografía reciente, en Italia el testimonio de las escritoras que vivieron ese período, viéndose directamente implicadas, no ha recibido el estudio merecido. Así, presentamos aquí un recorrido de la contribución de algunas de las escritoras más representativas del siglo XX.

Palabras claves:

Primera Guerra Mundial, Escritoras, Italia, Género.

\section{Abstract:}

Despite the fact that the active role executed by women in WWI society has been recognized by recent historiography, in Italy, the testimony of women writers who experienced this war period has not received all of the critical attention it deserves. Thus, this article introduces the contribution of some of the more representative women writers of 20th century.

\section{KeY WORD:}

Great War, Writers, Italy, Genre. 
"Il mondo non sa il cuore che esse hanno, nel loro tacito sacrificio" (Serao, 1916: 145)

Aunque son muchos los testimonios literarios femeninos de estos años convulsos, nos centraremos especialmente en las obras de las escritoras más representativas de la literatura italiana de la época, como Matilde Serao, Grazia Deledda, Sibilla Aleramo, Amalia Guglielminetti, Paola Drigo, Donna Paola, entre otras, e identificaremos las constantes estilísticas y temáticas que son comunes en la narrativa de guerra de estas escritoras.

Sin haber guerra, tampoco hay paz. Las escritoras ponen de manifiesto, en textos de variado estilo y cualidad, no sólo la dureza de la vida de la mujer durante la contienda: la soledad, el sacrificio y el sentido del deber y del auxilio, sino también el grito de libertad que sintieron muchas de aquellas mujeres que luchaban por conseguir su sitio en una sociedad patriarcal y machista, profundamente católica y conservadora, unificada recientemente, en la que todavía se dejaban sentir los ideales revolucionarios, pero que se fueron viniendo abajo, abatidos por la crisis de principios de siglo y la guerra que se estaba gestando.

Por primera vez, con la guerra, la mujer cobraba importancia, aunque su contribución se limitara a las tareas de maternage. Estas tareas y capacidades que se consideraron intrínsecas y obligatorias a y para toda mujer son el sacrificio, la capacidad de soportar, la obediencia, la fidelidad y la lealtad. Que haya prevalecido esta idea ha hecho que se vea reducido el interés y el estudio, relegando el papel de la mujer al mundo de la caridad y a un segundo plano. Además, encontramos mucha literatura propagandística, lo que ha desviado el interés por lo que ellas escriben, y esto es lo que ponemos en tela de juicio con este artículo, un tema que se debate continuamente entre el análisis del progresismo y del conservadurismo más radical, entre el feminismo y el antifeminismo.

Al mirar los hechos con la lupa del tiempo nos encontramos con ciertas ideas preconcebidas que nos pueden llevar a juzgar de forma superficial eineficaz el contenido de las palabras escritas por nuestras escritoras. Las escritoras aquí mencionadas tienen algo en común, y es la reivindicación de un verdadero y fundamental papel de un "ejército resistente" femenino: crocerossine y enfermeras, pero igualmente trabajadoras de todos los ámbitos, privados y públicos.

Por otra parte, esa mujer que tanto ha hecho por mantener la vida y el día a día durante la contienda, es la que siempre sostiene el peso. Franchi habla del peso de la nación, Serao o Donna Paola hablan del peso de la familia. Y para sostenerlo se necesita unidad. La fuerza de las mujeres unidas por una misma causa, ya fuese trabajar fuera de casa, ya fuese concienciar de la necesidad de ayudar, concienciar de la necesidad de autonomía o enviar ropas cálidas al frente para que hijos, hermanos, maridos y padres no pasaran frío. Como la guerra afectó a todas ellas, la experiencia del dolor y del luto las unió. Y esta unión es la que destaca en la narrativa estudiada. No se trata de una unión necesariamente feminista, en muchos casos, todo lo contrario, sino la unión movilizada y consciente en un contexto en el que ellas tienen que sacar adelante la vida de la nación y la vida de las familias.

La presión sobre las mujeres había sido enorme durante la Guerra de Libia, dirigida a alterar la naciente reivindicación femenina, temida también por la Iglesia, para sustituirla con un nuevo modelo inspirado en la sacralidad superior de la figura materna. Hay que pensar en la importancia del valor simbólico de la mujer en el período bélico (es necesario pensar en el binomio madre-patria y en la retórica propagandista que hacía necesaria la defensa de la guerra por parte de las mujeres, identificadas con la casa y la familia).

Con el estallido de la Gran Guerra, el sentimiento de culpa invade a las escritoras, sobre todo a las nacionales, como Ada Negri y Matilde Serao, culpa por la "terrorizzante grandiosità del dovere" (Citado en Gubert: 2013: 587) solicitado a los hombres. Creen que pueden no estar a la altura, pues ellas no van al frente. Esto origina posiciones radicalmente militaristas y cambios sociales. La movilización de la mujer se realiza ya por la situación de guerra y por ese sentimiento de culpa. Se fue afirmando cada vez con más fuerza el sentimiento de la Patria junto a la convicción de que la mujer debía socorrer a los hombres en el momento de la necesidad. Más tarde, sobre los años $20 \mathrm{el}$ acento se pone sobre una maternidad heroica al mismo tiempo que une a las mujeres, que cobra importancia a gracias a las asociaciones femeninas de apoyo a las madres y a las viudas y enaltecida después por el fascismo.

En dos poemas de Ada Negri encontramos los tres elementos fundamentales de la literatura femenina de guerra, que son, pues, la Patria como madre superior a todas las madres, que merece el mayor amor y el mayor sacrificio; el dolor por la muerte de cada soldado, pero un dolor que lucha entre la anulación en la masacre colectiva y el dolor individual y personal de la familia; y por último, el estoicismo obligatorio que se les pide a las familias, y en concreto a las madres y mujeres, pues la muerte de los hijos es "necesaria", un dolor que hay que padecer por un bien colectivo mayor: "Fra la guerra e la filosofía, la muraglia è caduta. Giacchè se vi è una filosofia necessaria in questo tempo crudele, è lo stoicismo" (Donna Paola, 1915: 157)

En el conocido poema "La madre" (1912), publicado durante la Guerra de Libia la figura materna está sometida a la campaña propagandística del momento y el hijo se convierte en el sacrificio supremo a la madre Patria, sin lágrimas. En el poema "Il soldatino ignoto" (Racconti e poesie), 1917, el sentido de la muerte y del dolor de la madre está encerrado en el nombre rimbombante de la Patria. En esta lírica emerge claramente 
la distancia entre las celebraciones públicas y la realidad íntima de las familias, de las madres de los jóvenes en la guerra: la contradicción del sacrificio materno por la Patria y el dolor de perder a su hijo, perfectamente retratada en los cuentos de la véneta Paola Drigo.

Esta escritura para infundir coraje y valor a las mujeres se repite en nuestras escritoras, notablemente en Matilde Serao y en Donna Paola: “Donne d'Italia, ci fu data una croce, la guerra: solleviamola, con coraggio, con forza; ed essa ci sembrerà un austero dono, se possa rendere più gloriosa la patria italiana" (Serao, 1916: 6). Sin embargo, es claramente propaganda, y la realidad es mucho más compleja que eso, pues he aquí la contradicción:

\begin{abstract}
dai diari e dalle memorie delle madri, emerge che molte di loro vissero una lacerante
contraddizione. Nella loro azione educativa, infatti, avevano promosso nei figli la passione per la patria; poste di fronte alla guerra vera, peró, si resero drammaticamente conto $\mathrm{d}$ per la patria; poste di fronte alla guerra vera, pero, si resero drammaticamente conto di
non riuscire più a sostenere e condividere gli ardori dei figli che si offrivano volontari. non riuscire piu a sostenere e condividere gli ardori dei figli che si offrivano volontari. A costo di sembrare egoiste o possessive, cercarono di sottrarre i figli al pericolo reale,
ad esempio facendo ricorso a conoscenze nei comandi militari. Molte lettere, pertanto denotano un forte scontro generazionale, fra madri tutt'altro che risorgimentali e figl denotano un forte scontro generazionale, fra madri tutt'altro che risorgimentali e figl
desiderosi di eroismo, di avventura e (forse) persino di maggiore libertà, rispetto alla tutela materna; oppure, ci troviamo di fronte a madri che ostentano in pubblico (e davanti al materna; oppure, ci troviamo di fronte a madri che ostentano in pubblico (e davanti al
figlio) calma, autocontrollo, serenità e patriottismo (accettando persino l'eventualità della morte in battaglia del giovane volontario), mentre in privato sono letteralmente distrutte e angosciate, di fronte al rischio dell'uccisione, oppure disperate e tormentate dai rimorsi, in caso di decesso avvenuto. (Feltri, 2016: 4).
\end{abstract}

En palabras de Anna Franchi en Il figlio della guerra: "In quel momento una passione lo trascinava. Andava verso il destino suo e della sua terra. Amava egli sua madre? Certo. Ma più forte ancora, dal profondo dell'io, sorgeva un amore infinito per una madre comune... Patria! Sublime madre nostra, il tuo volere sia fatto" (1918: 12).

Los escritos de Donna Paola ${ }^{1}$ y los de la Serao son los que más radicalmente establecen las funciones de la mujer en los tiempos bélicos y el comportamiento adecuado frente al luto, al dolor y al sufrimiento, y a la sociedad, justificándolos con los deberes de las mujeres para con las necesidades de la Patria y las expectativas que esta tiene de la mujer. En este contexto, ellas defienden la oportunidad que tiene la mujer de

1 La posición de Donna Paola es delicada con un recorrido poético y político complejo. En efecto, como su poética y su compromiso se habían ido desarrollando durante los años que transcurrieron después de la unificación italiana y con el movimiento por la emancipación de la mujer, desde é allá por el inicio de la guerra, "diversas fases con más fracasos políticos y desilusiones en su hace que logros significativos" (Valencia, 2015: 69). Esta frustración cambia de bando su compromiso. Así pues, la emancipación de la mujer para Donna Paola y Matilde Serao termina donde termina la guerra. Ambas hablan en contra de la emancipación de las mujeres fuera de la situación bélica: lo que prima es una visión de revalorización del papel que ha de jugar la mujer pero aún sin renunciar a la función tradicional que se le tenía asignada, quizás porque era consciente de su importancia social y de la poca capacidad del hombre en estos temas. Para más profundidad y un mayor conocimiento sobre Paola Baronchelli Grosson, conocida como Donna Paola, ver el reciente artículo de Valencia (2015: 67-76). demostrar su valor y su contribución. “Chi avrebbe mai sognato... che la guerra -cioè quel complesso di fatti e di attività che sembravano i più stranei alla capacità femminile - sarebbe stata di tutti gli eventi della vita nazionale, quello che più avrebbe messo in valore il contributo della donna?" (Donna Paola, 1915: 8). Por otra parte, Donna Paola analiza la sociedad y establece una serie de necesidades sociales y físicas en los ámbitos de la atención de las enfermeras, económico y social para los repatriados, y en la educación de los hijos de las mujeres que se marchan temporalmente para ayudar.

La mujer debe permanecer leal a los principios tradicionales más que nunca, incluso si sale a trabajar para compensar la balanza en las "actividades colectivas" públicas, su deber es ejercer este trabajo de la manera más eficaz posible y volver a casa, sin demostrar que la vida para ella sigue a pesar de tener al marido o al hijo en el campo de batalla. La vida, más que nunca, debe refugiarse detrás de la folla de la que habla Serao en su diario Parla una donna, la colectividad que encarna, unida y estoica, toda junta, ese dolor y ese horror de millones de rostros.

La posición claramente antifeminista de la Serao nos interesa para entender su literatura. El estudio de esta escritora demuestra que no solo la guerra cambia radicalmente la situación de Italia, sino también la eterna diferencia político-social entre el sur y el norte de la península². La mujer, para la Serao, siempre se confundirá con la folla, desaparecerá, será esencial, pero obligatoriamente invisible: "queste donne [...] compirono, per amor patrio ardentemente sentito, il più tacito e il più puro fra $\mathrm{i}$ sacrifici, rientrando nell'ombra, confondendosi nella folla" (Serao, 1916: XIII). Tanto para Donna Paola como para Matilde Serao, la mujer que acepte el deber del sacrificio será, para la Patria, tan digna como el marido, el hijo o el hermano, y recibirá la gratitud pública y la admiración familiar. "La funzione della donna in tempo di guerra, si inizia dunque con una grande opera morale: sapersi sacrificare senza pianto, per infondere coraggio al combattente" (Donna Paola, 1915: 6):

Voi che non volete soffrire, voi che non sapete soffrire? Darete voi questo malo esempio, a coloro che vi amano, ai vostri amici, ai vostri servi? Sarete una pietra di scandalo, perché non sapete e non volete soffrire? O diverrete una creatura d'indifferenza e di debolezza? Aumenterete, così, la pena di tutti quelli che vi circondano, invece di esser, voi, il centro della loro sublime rassegnazione e della loro sublime pazienza, come è il vostro dovere di donna [...]? (Serao, 1916: 4-5).

2 "La posizione della Serao è del tutto condizionata dalla società nella quale si trova a operare, quella società meridionale che stava progressivamente perdendo terreno, sul piano economico come su quello culturale. In questo contesto tutte le forme di radicalismo [...] non avevano alcuna probabilità di essere comprese. Una società bloccata nel proprio sviluppo e respinta verso la dimensione rurale non poteva, infatti, riconoscerci nelle rivendicazioni di una borghesia urbana avviata verso la società industriale" (Briganti, 1988: 193).

Revista Internacional de Culturas y Literaturas, abril 2017

ISSN: 1885-3625 
El elogio de la asistencia femenina durante la guerra se acompañó del auspicio de una rápida vuelta de las mujeres a la esfera de lo privado y a su deber eterno: ser madre es, para la mujer "in tutti i tempi e in tutte le nazioni, il dovere dei doveri" (1916: 137)

El mensaje moralista impregna todos sus artículos: los puntos fuertes de la moral de Serao son la virtud del silencio y de la resignación cristiana: "Chi sa amare, sa soffrire, sa sopportare, sa attendere" (1916: 203). Moral desprovista de todo análisis real, órdenes que no dejan espacio a la reflexión sobre el nuevo papel de la mujer: "Dovete lavorare la lana, perchè i soldati d'Italia non muoiano di freddo. Non pensate ad altro. Non fate altro. Lavorate" (1916: 187).

La lana nos lleva, ya lejos del fervor patriótico visto hasta ahora, a las prosas, entre los escritos literarios bélicos más interesantes, que van desde el retrato a la novela, pasando por los cuentos o novelle, cuyas representaciones leen la guerra en la incidencia sobre la vida cotidiana de la mujer, elemento nuevo (a veces activo) que entra en el orden constituido y lo subvierte.

\section{La mia parola arriva tra uno scoppio e l'altro di granate, nella ridotta blindata d'acciaio, in mezzo alle nevi. Gli spira alito di fiori e di spiaggia, gli è sostanza di silenzio e di trasparenza, iridescenza di fantasia, comando di speranza.... Diletto e balsamo. Bei vocaboli antichi e nuovi. La vita è una sfolgorante fola. Io sono una} rondine e con l'ala accarezzo una fronte di condottiero (ALERAMO, 1920) ${ }^{3}$.

En la prosa "Lavorando lana", del 1915 (del volumen Andando e Stando) se encierra la totalidad de lo que pensaba Aleramo sobre la guerra: "Pareva, in principio, allo spirito impavido, che nessuna attività vitale verrebbe mai per la guerra sospesa. Invece, ecco, non riesce altro che a lavorar lana" (1920). La tranquila sencillez del trabajo de la lana actividad tan difundida entre las italianas comprometidas en preparar indumentaria cálidas para mandarlos al frente, se convierte en el pretexto de una reflexión genera sobre la vida en aquel tiempo suspendido, sobre el arte, la mujer, y sobre ella misma:

E tutto di questo desolato squallore io avevo già provato, nei tempi che si chiavan di pace: niente m’è nuovo. Se non la materialità, la ferinità della causa [...]. Così di pace: niente m'e nuovo. Se non la materialita, la ferinita della causa [...]. Così volta davvero, sentono ora soltanto, e soltanto per riflesso, che cosa veramente significhi vivere in pericolo! L'esercizio spietato di tutti i miei anni non è ancor sufficiente ad impedir ch'io sia dilaniata per tristezze che ritornano, identiche come le stagioni: ma tutte costoro che, di repente devon sbarrar gli occhi dinanzi alla crudeltà d'un dato destino, d'una data epoca: impreparate - nessun miracolo di reincarnazione s'era manifestato in esse e le aveva sferzate sin dalla nascita; devono staccarsi dal figlio dall'amante dalla quiete dal sonno; e le sopracciglia s'alzano interroganti vane; $\mathrm{v}^{\prime}$ ha tra queste donne di quelle che non han mai saputo dormire sole in

3 Todas las citas de este párrafo y los siguientes sobre Aleramo pertenecen al libro que estamo estudiando, Andando e stando (1920) pero la versión consultada es una versión electrónica y carecía de una numeración formal y clara, por lo que muchas de las citas se encuentran sin página. sostare in posti sconosciuti, esser trattenute da piantoni inflessibili, giunger troppo tardi... Diventan simboli. Ecco l'ironia (1920: 188).

Para Sibilla, las mujeres no viven el verdadero peligro, pues no combaten: lo viven "soltanto per riflesso" (1920), pero aún así, este reflejo causa tanto daño, que las mujeres se convierten en símbolos de la guerra, la destrucción, el sufrimiento y el sacrificio. Deja la propaganda a un lado y muestra el lado más humano de la guerra en "Lavorando Lana":

Li amavo. Poveri. Vili. Con tanto freddo nei cuori. Con tanto terrore delle lagrime. $\mathrm{E}$ più ancor delle maschere sarcastiche eran tristi a vedere le corazze d'orgoglio.

E più ancor delle maschere sarcastiche eran tristi a vedere le corazze d'orgoglio.

$\mathrm{Ma}$, quando l'ora suonava, foss'anche una sola, si scioglevan tra le mie braccia, come bimbi tra quelle della madre al buoi. Santità del pianto virile, della virile miseria che si confessa (1920).

La posición de Sibilla Aleramo es la más compleja: no está a favor de la intervención, parece que se acoge a la posición de algunas mujeres socialistas, pero aun así tampoco condena la empresa bélica. "Non so [...] che cosa io stia precisamente scrivendo. Io che non posso esaltar la morte nel carnaio guerresco. E che tuttavia non impreco" (Aleramo, 1920: 175)

Las turbulentas páginas de Andando e stando, del año 1920, nos conducen a las impresiones de viajes publicadas dispersamente en periódicos, (estaba cumpliendo en esta época, 1915, su viaje estético entre París, Asís, Florencia y el sur de Italia), recogidas después en ese volumen: se trata de algunas de las prosas más fuertes de Sibilla y más vivas sobre el tema bélico, una guerra que se implanta en la vivencia cotidiana de una mujer sola que todo observa y analiza con atención. La guerra lo impregna todo, se mezcla con las viejas costumbres y no deja lugar para nada más; se ve en los imprevistos e inesperados gestos patrióticos (en el trabajo, en los mítines o en el cinematógrafo) de la población que está en casa: las mujeres que trabajan la lana para el frente, los muchachos que se mofan del Kaiser, los campesinos que escuchan asustados el mitin en una lengua que no comprenden pero que enciende en ellos el orgullo nacional. "È uno sguardo penetrante ma obliquo rispetto alle descrizion patriottiche di altre scrittrici. La guerra emerge tanto più lacerante quanto più è un elemento straniante nell'antica vita delle popolazioni italiane" (Gubert, 2013: 595).

Aquí están dos de los artículos más importantes de la época (1911) sobre la guerra de Libia: "Apologia dello spirito femminile" y "L'ora virile", dedicado a la empresa bélica y en donde, por primera vez, se afirma públicamente la necesidad de contar con un tipo de apoyo de las mujeres en la guerra, a través del silencio. Con este silencio, según Aleramo, "le sorelle realizzavano il proprio lavoro" (Gubert, 2013: 594). Parece que el silencio, de nuevo, crea consenso entre las escritoras: el dolor producido por 
la guerra es tal, que se posicionan en muchos casos a favor del silencio de la mujer y su resignación, comportamientos escondidos tras el miedo de crear una situación aún más caótica en la sociedad del momento y a perder la fuerza que estaba cogiendo el movimiento feminista. El apoyo a la patria se cumplía, por lo tanto, a través del silencio, por parte de las escritoras, con la ausencia de palabras vanas, rescatando así la concepción de la mujer del imaginario masculino que las quería frágiles y llorosas (Gubert, 2013: 595).

En la sección "Volti e destini" la guerra sirve de fondo para los retratos de los amigos muertos, como el de la "sorella spirituale" (Gubert, 2013: 595) "Alessandrina Ravizza", figura de referencia de la emancipación femenina y del mundo de la asistencia. De Scipio Slataper hace el retrato más breve e intenso de un caído en batalla: "S’è fatto ammazzare per attestare disperatamente la propia unità morale" (Aleramo, 1920: 170172). Y añade:

\section{Se spiegare giovasse a consolare. Se il dire alla giovine donna che piange l'amato e ha fra le braccia un bimbo nato ieri, il dirle che il suo uomo si sarebbe spezzato ugualmente sul limitare di giovinezza anche senza l'assurdo di questo moto tellurico che dura ininterrotto da mesi, se il dirle questo potesse lenirle il dolore, placarle la santa elementarità del pianto (1920: 173).}

En "Frate Ferro a Renato Simoni" aparece reflejado el patriotismo a través de las reuniones en el teatro para mostrar a los niños proyecciones de guerra del frente francés. Y cuando llega la tarde y la noche, todavía hay gente para llenar la sala: viejos señores, viejos obreros, otros chicos y algún cura, y mujeres, muchas mujeres. El dolor es colectivo y todo se convierte en un "irresistibile invito al pianto e al canto in comune". Sibilla humaniza sus discursos, trayéndolos al plano cotidiano y problemático de la tensión entre el significado de ser hombre o mujer en una sociedad en guerra. No podemos separarnos de su feminismo para analizar y señalar su escritura de guerra.

Sibilla intenta darle a cada persona y a cada sentimiento su lugar: hay resignación, pero una resignación consciente del papel y del sufrimiento de la mujer, que no lo niega, que no lo enaltece ni lo rechaza: "Questo pianto che nessuna ostenta, perché la nazione dovrebbe ostentare d'ignorarlo?". La guerra va más allá de una empresa de hombres o de mujeres, "E l'ora di guerra non è né virile né femminile, è soltanto un'ora necessaria, come tutte le ore della vita, ove il bene si fonde al male, ove l'eroismo poggia sulla viltà, e la speranza nasce dalla sciagura".

"Patria civiltà diritto: mondo natura forza": esa es la simbiosis que se da en la guerra, y esta, entre naciones, entre razas, es una creación del hombre, exterior e interior. La mujer la acepta, como él, pero es más infeliz que él, pues no participa en ella, porque en vez de derramar su propia sangre, tiene que ver cómo sus hijos, sus hermanos y maridos la derraman. El dolor de la mujer viene de su tremenda generosidad y amor. La única manera de que la mujer se exalte con la guerra es con el dolor y con la imaginación de los campos de batalla, nada más. Y, aunque no consiga superar ese dolor materno, no grita y calla. La guerra se cuela entre las grietas de las puertas de cada casa, de cada familia y muta el día a día de forma personal y colectiva. Así, las víctimas civiles, ajenas a la contienda, representan la unión más importante de esta época.

Debemos hacer referencia al premio Nobel Grazia Deledda, a quien se le reprocha su silencio de frente al hecho bélico. Il ritorno del figlio guarda estrecha relación con nuestro tema: la historia no siempre resulta plausible, es patética, desarrollada en una manera compleja y con una ambientación vaga. Se centra en el proceso psicológico vivido por una madre "alienata dalla vita dopo la morte in guerra del figlio (un raro riferimento all'esperienza bellica appena conclusa). Solo quando le viene portato in casa un bambino, trovato dal marito abbandonato in una strada di campagna, sente rinascere la sua capacità di amare" (Deledda, 1996: 7).

Il ritorno del figlio es el único cuento de toda su extensa producción que tiene como trasfondo la guerra y el dolor que esta provoca: es la historia de una familia de un pueblo, no sabemos cuál, que tiene sólo un hijo y lo pierde en el campo de batalla. Pero el destino les traerá de vuelta un bebé abandonado en mitad del camino principal del pueblo al que decidirán cuidar como a un hijo. Aunque el argumento es simple $a$ priori y parece cándido, la psicología humana se pone de manifiesto en todo el cuento, mostrando el lado más detestable del ser humano y la hipocresía del egoísmo y la moral de la pequeña burguesía. Asimismo, la historia pone de relieve que no solo la guerra deja víctimas, sino que las mismas son crueles y sólo buscan su propio bien, por lo que, al mismo tiempo, son también víctimas de ellos mismos. La guerra les ha arrebatado al hijo, ¿pero y ellos?, ¿dónde quedan los sentimientos?, ¿qué significaba el hijo antes para ellos? "In fondo cos'è che si ama nei figli? Noi stessi, sempre, fino a che siamo morti o che loro sono morti" (Deledda, 1996:47).

La tensión que supone asumir la culpabilidad y el castigo o hacer responsable a Dios se manifiesta en toda la historia: "Gli uomini sono guidati da Dio [...]. No, no. Non è Dio a volere questo cose orribili. Me l'hanno portato via gli uomini, me lo hanno ucciso gli uomini" (Deledda, 1996: 47). La guerra le sirve para hacer entrar en juego la psiche humana y el conflicto en uno mismo y en una familia. Todos son víctimas, y la Patria o la colectividad no aparecen por ningún lado. El odio lo impregna todo: “Ma non voleva muoversi, no: anche perché sentiva un odio sordo contro il brigadiere, che per lui era uno di quei feroci personaggi che tutti in blocco rappresentavano la Forza mostruosa che le aveva tolto il figlio di casa per buttarlo nei campi della morte" (Deledda, 1996: 44). 
La estática del pueblo y la historia del cuento que, como decíamos antes, permanece fuera de la Historia y del contexto, se mantiene en la periferia de la narración que aparece representada en la familia. La guerra es el marco y la explicación de la muerte del hijo que, por otra parte, perfectamente podía haber muerto de otra forma y la historia no cambiaría nada. Lo importante es el odio que hace nacer. En los personajes no queda espacio para la posibilidad de cambio. El pasado los ha curtido así y, aunque mortales, se funden con el escenario de objetos: "Gli oggetti non muoiono e non moriranno" (Deledda, 1996: 37).

La guerra existe porque los hombres no tienen corazón, de lo contrario no existiría y el rencor es la mejor manera de vivir para el protagonista: “Non bisogna aver pietà Ne hanno avuta, gli altri, con noi? [...]. È che proprio bisogna non aver pietà né amore si vive meglio!" (Deledda, 1996: 38). Deledda nos muestra aquí un hombre que ha elegido el deber de vivir a través del rencor, que, o se ha equivocado de deber o es el determinismo de la guerra y de ese contexto rural. En contraposición, la mujer, esperaba, silenciosa, egoísta en su terrible dolor, pues tampoco ella quiere saber nada del bebé cuando el marido lo lleva a casa. La viuda espera a su hijo muerto: "tutta la sua attitudine era di chi aspetta pur sapendo che la sua attesa sarà lunga e forse vana" (Deledda, 1996: 28). Por otra parte, también podemos ver a una familia "muerta" porque ha muerto el hijo: la denuncia de las miles de muertes de soldados, que suponían la "muerte" de las respectivas familias.

Pero estas historias no siempre interesaban a los contemporáneos, que no apreciaban los cuentos y novelas cuando se alejaban de la propaganda. Por ejemplo, Lipparini, a propósito de Le ore inutili, de Amalia Guglielminetti, escribía que "si è buttata ad una superficialità esasperante, buona forse per i cervelli vuoti di molte donne" (Citado en Gubert, 2013: 597)

Los cuentos de Amalia Guglielminetti, publicados en 1919, alinean y exponen los principales actores del fervoroso imaginario producido por la sociedad italiana durante la guerra, revelando a veces, a través de la ironía, el vacío de estos. Todos los personajes, mujeres y hombres, civiles y también soldados, aparecen en una dimensión siempre doméstica. Con una representación amarga y sin retórica, la escritora busca la vida en los pliegues, imperfectos, de las relaciones amorosas, familiares y sociales.

La guerra, retratada en Le ore inutili, es un evento que suspende las convenciones normales entre los individuos, dejando paso aparentemente a nuevas experiencias y oportunidades que, sin embargo, bajo el trazo rápido y contundente de la escritora, indefectiblemente terminan en decepción, absorbidas por un orden que no muta nunca: en La Salvatrice, la felicidad permanece inalcanzable, rompiéndose bajo el sentido del deber; la defensa de la familia obliga a ceder ante la hipocresía hasta el sacrificio de la familia misma o de otros (Scherzi di guerra); incluso sitúa como protagonista una figura que se encuentra entre las más censurables de la época: uno al que por un defecto físico (en este caso por una joroba) se le margina y se le ridiculiza, considerado culpable implícitamente por no estar en el frente combatiendo. Vivir como hombre significaba ser soldado y tener una mujer, así que el protagonista de Il sovrappiù se suicida entre "la musica e le grida" (Guglielminetti, 1919): la guerra, la propaganda que tapa la realidad, la patria, los deseos de la mayoría ciega, que se llevan por delante todo y a todos.

Además encontramos un cuento (La verità) con el mismo argumento de una historia del escritor Gozzano, Gli occhi dell'anima: mientras que el marido está en el frente, su joven y bella mujer sufre un accidente y se queda con la cara desfigurada. Entonces teme ser rechazada y le esconde a su esposo la verdad hasta que él es herido y la llama al hospital militar. En ese momento, dividida entre el horror y el alivio, descubre que él se ha quedado ciego. La batalla personal contra el disfraz moral encuentra el culmen en el cuento L'intrusa, donde se desmonta el mito de la solidaridad natural entre mujeres durante la contienda, en una historia sobre el amor conjunto, pero no compartido, de dos figuras emblemáticas, madre y esposa, por un soldado que muere.

Aquí se conjuga el doble dolor femenino: de madre y de amante. El estoicismo emerge de nuevo como actitud necesaria, egoísta y protectora, pues no todo era fraternidad y solidaridad entre todas las mujeres. Guglielminetti no muestra el idealismo en el sufrimiento de las mujeres, sino que parece que se compita por el dolor del egoísmo materno: "Ora essa scenderà. Ora io rimarrò sola col mio dolore senza che codesta importuna compagna mi osservi e mi commenti. Ora io potrò finalmente piangere, gettarmi sul divano e singhiozzare e gemere e non più comprimere dentro di me questo male che mi torce il cuore" (Guglielminetti, 1919).

El luto es la experiencia que acompaña y une a las dos protagonistas de este cuento, empeñadas en permanecer distanciadas. Y, de igual modo, la experiencia que acompaña en modo más o menos directo, a todas las mujeres durante la Primera Guerra mundial. Esta dimensión de la guerra determina los comportamientos públicos y privados de las mujeres. El luto privado asume la forma de ritual público de sublimación del sacrificio de los soldados y se convierte en elemento esencial de un proceso de “monumentalización" de la guerra (Molinari, 2008: 11) y convierte a las mujeres en sujetos activos de la guerra.

Por otro lado, gracias a la guerra, en La via ritrovata, un joven encuentra su vocación:

[...] dinanzi agli occhi sbalorditi della marchesa Saveria quell'uomo inginocchiato che le baciava la mano, Jacopo, il monaco della più rigida clausura, apparve vestito d'una divisa d'ufficiale segnato al braccio di una rossa croce [...]. - Mi hanno chiamato ed eccomi qui, pronto a tutto. Soccorrerò i feriti, benedirò i morenti, affrenterò io stessto la morte [... E forse, nonna [...] forse, nonna, trovero finalmente la mia via. 
Jacopo encuentra así el sentido de su vida, y la conciliación viene sola. Matilde Serao, el diario de guerra, dice: "E se la guerra cambia i destini di migliaia di persone, ogni tanto essa lo capovolge, questo destino" (1916: 155).

En La salvatrice Guglielminetti muestra claramente el horror físico y psíquico de la contienda a través de las heridas de un soldado.

I suoi occhi sembrano quelli d'un convalescente, ma d'un convalescente che non abbia voglia di guarire [...]. - Sono uscito ieri l'altro dall'ospedale militare, ma non sono ancora guarito. Non potrò mai guarire [...]. Una scheggia di granata qui, nella gamba destra, e nel cervello uno smarrimento, un orrore, una visione così terribile che mi ha istupidito per sempre.

Destaca igualmente la figura de la mujer como salvadora del ánimo y de la mente del hombre, del soldado, que lleva demasiado tiempo sumido en la pesadilla constante y en el shock de la guerra. Salvadora porque ella ha permanecido ajena a la barbarie del frente, o la ha sufrido de otra manera y, por tanto, tiene ese poder rejuvenecedor a través del amor.

Para terminar, del Piamonte saltamos al Véneto, región que sufrió en el propio territorio la guerra, con Paola Drigo ${ }^{4}$, que es quizás quien en algunas historias presentes en su segunda publicación, Codino (1918), representa mejor la condición de la mujer de orígenes humildes durante la guerra. Lejos tanto de la retórica corriente como de la mundanidad de Guglielminetti, afronta en sus cuentos los "problemi di vita" (Citado en Gubert, 2013: 598).

La guerra es narrada en dos cuentos ejemplares de la tercera parte de Codino, su obra más conseguida: La zia e Tonet ell volontariato di Torquemada. A través de la contemplación severa y dolorosa al mismo tiempo, calmadamente irónica, del destino ineluctable que gravita sobre el ser humano, se asiste a la "sommessa esaltazione dell'eroismo bonario e casalingo che trasforma una vecchia zitella e un cocco di mamma in autentici eroi patriottici" (Citado en Gubert, 2013: 598). Heroísmo ensombrecido por la muerte, por la mutilación, que deja en el lector sinceras dudas sobre la recompensa real de ese coraje. La ironía se deja ver también en la esquematización de los personajes, reducidos a personalidades bien definidas, con intereses e instintos simples, con las que la escritora jugará con la pérfida intromisión del destino que vuelca, en un instante, las vidas y los caracteres de la pobre gente.

Esto se ve bien en Il Volontariato de Torquemada, donde Drigo hace emerger las contradicciones profundas que hay en el ánimo de los italianos frente a un patriotismo más impuesto que deseado: el padre se debate entre el remordimiento por haber

4 Al contrario de Serao o Negri, consideraba imposible, en virtud de la fascinación ejercida por un padre patriota, culto y anticlerical, creer en la Providencia divina, y edificar por tanto los destinos (también los de sus personajes) según una visión metafísica o religiosa. enviado al hijo al frente y el temor que por debilidad de carácter, este no se gane el honor que reclama la propaganda nacionalista. En este tormento, el viudo asume los trazos maternos, pero de una madre insegura y frágil, llorona, opuesta a la imagen de la madre valiente y orgullosa que sacrifica el propio amor puro por la patria. El resultado aparece así tragicómico y todo se vuelve complejo en el análisis psicológico de Drigo. La unión crea fuerza, pero también destruye. El final de la historia es destacable: el héroe realista que acepta su deber, que lo realiza y que continúa siempre fiel a sus principios: “- Non preoccuparti, papà... Sono stato ferito... sono stato ferito... volentieri" (1918: 122). Toma se alista como volontario, y lo que hace al salvar al oficial es, efectivamente, volentieri.

En La zia e Tonet, la vieja tía y el sobrino cojo, Tonet, viven en un pueblo junto al Canal del Brenta, y la tía tiene una pequeña taberna, donde continúa trabajando por inercia. Cuando comienza la guerra, los soldados que acampaban por la zona del Brenta van cada día a la taberna, y esas tropas representaban lo que para Teodora era la guerra:

quella cosa orribile mostruosa iniqua inumana, repugnante alla sua anima mansueta come un castigo di Dio, come un flagello del demonio; dover toccarla con mano, averla sotto gli occhi; incoraggiarla, servirla, personificata in quei soldat che sedevano alla sua tavola, che bevevano nei suoi bicchieri, e parlavano ridendo di bombe, di granate e di mitraglia.... Oppure discutevano.... Sì! discutevano sui mezzi migliori per massacrare la gente (1918: 79-80)

Y así es como Tonet cobra vida y se convierte en un despierto y ágil ayudante de ellos, emocionado y entusiasmado por las historias de la trinchera y la aventura: "Fino ad allora il bimbo aveva vissuto una vita oppressa e senza gioia [...].Ora, appariva trasfigurato. Quella gioventù, quella gaiezza, quella corrente viva e sana improvvisamente apparsa nella sua vita, l'avevano elettrizzato" (1918: 78-82). Tonet salva a los soldados de un espía: no todo es fuerza y destreza militar, la astucia de un joven cojo puede hacer tanto o más que unos hombres cargados con sus fusiles por la guerra y por los soldados. Es así como Drigo nos muestra siempre el otro lado de la sociedad.

La escritora véneta nos sumerge de lleno en la realidad de la guerra en su tierra a través de historias personales, íntimas y cotidianas, que rompen los esquemas de la literatura femenina de guerra y que trascienden las fronteras del argumento al retratar la sociedad rural de la mujer soltera y tímida, el joven cojo o el pequeño burgués que se deja aplastar por la sociedad y manda al hijo a la guerra.

\section{REFERENCIAS BIBLIOGRÁFICAS}


Aleramo, S., Andando e stando, Firenze, Bemporad, 1920.

Arslan, A., "Ideologia e autorappresentazione. Donne intellettuali fra Ottocento e Novecento", Svelamento. Sibilla Aleramo: una biografia intellettuale, Milano, Feltrinelli, 1988, pp. 164-176.

Briganti, A., "Matilde Serao: un profilo", Svelamento. Sibilla Aleramo: una biografia intellettuale, Milano, Feltrinelli, 1988, pp. 188-198.

Buttafuoco, A. (1988). “Vite esemplari. Donne nuove di primo Novecento”, Svelamento. Sibilla Aleramo: una biografia intellettuale, Milano, Feltrinelli, 1988, pp. 139-163.

Curti, D., "Quella che va sola: Amalia Guglielminetti", Svelamento. Sibilla Aleramo: una biografia intellettuale, Milano, Feltrinelli, 1988, pp. 199-207.

Deledda, G., "Il ritorno del figlio", Novelle, Volume 4, Nuoro, Ilisso, 1996.

Donna Paola, P. B., La funzione della donna in tempo di guerra, Firenze, R. Bemporad e Figlio Editori, 1915.

Drigo, P., Codino, Milano, Treves, 1918. Internet. 16-07-2016. <http://www.liberliber.it/ mediateca/libri/d/drigo/codino/pdf/codino_p.pdf>

Feltri, F. M,. “Guerra e identità di genere in Italia", L'Italia nella Grande Guerra, 2010. Internet. 16-07-2016.

$<$ https://seieditrice.com/chiaroscuro/files/2010/03/V3_U2-ipertestoA.pdf $>$

Franchi, A., Il figlio della guerra, Milano, Treves, 1918.

Gualtieri, A., “La Grande Guerra delle donne, 1914-1918”, Lagrandeguerra.net., 2009. Internet. 19-07-2016.

$<$ http://www.lagrandeguerra.net/gggrandeguerradonne.html>

Gubert, C., "Cronache dal fronte domestico. Le scrittrici italiane e la grande guerra", AUSENCIAS. Escritoras en los márgenes de la cultura, Sevilla, Arcibel, 2013, pp. 585604.

Guglielminetti, A., Le ore inutili, Milano, Treves, 1919.

Molinari, A., Donne e ruoli nell'Italia della Grande Guerra, Milano, Selene, 2008.

Negri, A., Esilio, Milano, Treves, 1914.

Racconti e poesie della Grande Guerra. Piccola scelta a cura di Maurizio Vitali. Internet. 19-07-2016.

Serao, M, Parla una donna. Diario femminile di guerra. Maggio 1915 - Marzo 1916, Milano, Treves, 1916.

Thébaud, F. “La Grande Guerra: età della donna o trionfo della differenza sessuale?", Storia della donna in Occidente, Roma-Bari, Laterza, 1992, pp. 25-29.

Tortoreto, A., "Pagine di donne italiane al tempo della Grande Guerra", Al tempo della Grande guerra. Due saggi e un diario, Milano, Edikon, 1968, pp. 11-30.
Valencia, M. D., “Mujer y nación en los escritos bélicos de Paola Baronchelli”, Estudios Románicos, 24, (2015), pp. 67-76.

$<$ http://www.tracce.it/detail.asp?c $=1 \& p=1 \& d=43 i 082>$ 